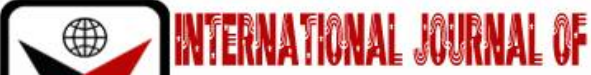

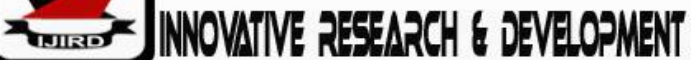

ISSN 2278 - 0211 (Online)

\section{Factorial Analysis of Maternall Mortality Audits: A Case of Migori County Referral Hospital, Kenya}

Ochieng Odhoch
Chief Executive Officer, Department of Medical Services, Migori County Referral Hospital, Kenya
Noah Adera
Biostatistician, Departments of Data Management, Phester Kenya, Kenya
Arthur Oyugi
Health Records Information Officer, Department of Health Records and Information,
Migori County Referral Hospital, Kenya
Lilian A. Ogola
Midwife, Department of Maternity Ward, Ambira Sub-County Hospital, Kenya
Prossy Wanyana
Carlmera Ochola
Medical Officer, Department of Newborn Unit, Mulago National Referral Hospital, Uganda
Chedical Officer, Department of Maternity Ward, Kehancha Sub-County Hospital, Kenya
Chester Kolek
Clinical Pharmacy Resident, Department of School of Pharmacy, University of Nairobi, Kenya

\begin{abstract}
:
Background: Maternal mortality is a global health concern especially in developing countries with $99 \%$ of all maternal deaths. Kenya reports a mortality rate of 342/100000 with majority of these occurring in northern and western parts of the country with Migoricounty recording annual maternal mortality rate of 673/100000. As part of Sustainable Development Goals, the target is to reduce the global maternal mortality ratio to below 70 per 100000 live births. Maternal death reviews are globally accepted as a means of mitigating against avoidable maternal deaths. Kenya adopted and rolled this in 2004. The efficiency of this intervention in major referral facilities remain unmonitored and undocumented.

Objectives: Major goal of this study was to examine the effect of maternal death review on maternal outcomes with specific aims being: To determine the prevalence and compare the trends of maternal mortality over two years, identify factors contributing to maternal mortality and to examine and compare the trends of factors leading to maternal mortality between the two years in the referral hospital.

Methodology: Data on all audited maternal deaths that occurred in Migori County Referral hospital between 2018-19 were abstracted from District Health Information system and analyzed using excel version 2007. Both descriptive and inferential statistics were used. We used both chi-square test and test of difference for proportions with CI interval set at $95 \% p=0.05$ to test our null hypotheses.

Results: Total of 36 maternal deaths were recorded during the study period with 16 (329/100000) and 20 (469/100000) occurring in 2018 and 2019 respectively. We found a significant association between parity and maternal mortality, $p$-value $=0.0027$ and a significant association between gestation and maternal deaths, $p$-value $=0.0006$.

Conclusion: Maternal death reviews has not led to reduction of maternal mortality in the referral hospital.
\end{abstract}

Keyword: Maternal mortality

\section{Background}

Maternal mortality remains a global health concern. Low- and middle-income countries are the main sufferers of this serious problem. World Health Organization reports that $99 \%$ of the global maternal deaths occur in developing countries.(Bale, Stoll, \& Lucas, 2003). From 1990 to 2015, the global maternal mortality ratio declined by 44\% -from 385 deaths to 216 deaths per 100000 live births according to UN interagency estimates.(WHO, 2015.) .Between 2016 and 2030, as part of the Sustainable Development Goals, the target is to reduce the global maternal mortality ratio to less than 70 per 100000 live births.(WHO, 2020). Maternal death reviews are globally accepted as a means of mitigating against avoidable maternal deaths. Review of maternal deaths assists in identifying important Quality of Care problems. In addition to identifying obstetric causes, these maternal death reviews shed light on why women are dying by identifying 
contributing and often avoidable factors and help to discover important shortcomings in care and weaknesses in organization and provision of health.(Hofman \& Mohammed, 2014).Facility-based MDR is defined as a "qualitative, indepth investigation of the causes of and circumstances surrounding MDs occurring at health facilities". The main purposes of MDR are to answer the question "why did this woman die?" to initiate action to solve identified problems, to improve QoC, and to save lives in the future. For those taking part, it is a valuable learning experience and each death tells an important story of what went wrong and what could have been done better.(Hofman\& Mohammed, 2014). World Health Organization recommended that all countries that had not established maternal death audit systems should do so without delay to help reduce maternal deaths.(Bale et al., 2003). Kenya has made great strides towards improvement of maternal outcomes. In 1990 the average MMR was 687 against 510/100000 live births in 2015 translating to annual rate of reduction of $1.2 \%$ which is lower than 5.5\% target.(WHO, UNICEF, UNFPA, World Bank Group \& Group, 2015). Kenya is among the 10 countries that comprised 58 percent of the global maternal deaths in 2013, contributing 2 percent of these deaths.(Koltai\& Schieman, 2015). The greatest challenge to reducing maternal deaths in Kenya lies in counties where maternal deaths are above the national average. These counties include Mandera, which has the highest mortality, at 3,795 deaths per 100,000 live births, followed by Wajir and Turkana, which have maternal mortality ratios of 1,683 and 1,594 respectively. (Koltai \& Schieman, 2015).Nyanza region contributes to higher MMR of 546/100000with Migori County reporting $673 / 100000$ per live births.(Koltai\& Schieman, 2015). The use of MDR guidelines was rolled out in Kenya as a tool in prevention of avoidable maternal deaths. It's unclear whether this intervention has had impact in its intended goal. My central hypothesis is that MDR has not led to improved maternal outcomes in Migoricounty referral hospital. To test my hypothesis, we conducted a retrospective cohort study on factorial analysis of maternal mortality audit reviews: a case Migori county. The ultimate goal of this study was to identify gap between audited maternal mortalities and recommendations made during audits with specific aims being: To determine the prevalence of maternal mortality in the referral hospital over two year, identify factors contributing to maternal mortality in the referral hospital, examine and compare the trends of factors leading to maternal mortality between the two years.

This study was conducted at the Migori county referral hospital and took the form of retrospective cohort design involving retrieval and review of past hospital records on maternal death audits for the years 2018 and 2019.This was managed and analyzed using excel version 2007. Continuous variables and effect factors will be presented as means and standard deviation while categorical and ordinal data are presented as percentages, or frequency distributions.

Permission to access this data was granted by the office of director medical services, Migori County.

The ultimate goal of this study was to identify if MDR has led to reduction of maternal deaths in referral hospitalwith specific aims being:

- To determine the prevalence and compare the trends of maternal mortality in the referral hospital over two years

- To identify factors contributing to maternal mortality in the referral hospital

- To examine and compare the trends of factors leading to maternal mortality between the two years.

\section{Methodology}

\subsection{Study site}

The study was conducted in Migori county referral hospital.

\subsection{Study Design}

This was a retrospective cohort study involving retrieval and review of all audited maternal deaths.

\subsection{Sample Size}

This was a census as all records of maternal death audits were reviewed.

\subsection{Data Collection}

Data on audited maternal deaths were abstracted from DHIS and further information were gotten from focused group discussions.

\subsection{Data Analysis}

The data was managed and analyzed using Excel Version 2007. Both descriptive and inferential statistics were used where data was presented in Continuous variables and effect factors were presented as means and standard deviation while categorical and ordinal data was presented as percentages, or frequency distributions. We used chi-square test and test of difference for proportions with $\mathrm{CI}$ interval set at $95 \% \mathrm{p}=0.05$ to test our null hypotheses.

\subsection{Ethical Consideration}

Permission for this work was obtained from the director Migori county health department.

\section{Results}

\subsection{Introduction}

Total live births for 2018 was 4864 with 16 maternal deaths in the same year translating to maternal mortality ration of 329/100000. In the subsequent year, 2019, there was a reduction in live births by 600 to 4264 with 20 maternal 
deaths translating to MMR of 469/100000. This MMR are higher than the national MMR of 342/100000 though lower than the county's which is at 673/100000.

In 2018, 913 (18.8) mothers delivered via caesarian section compared to 2019 that had 716 (16.8). Breech deliveries accounted for 53 (1.0) in 2018 with 55 (1.3) in 2019 respectively.

\subsection{Perinatal Survival Analysis for Babies Whose Mothers Died}

We found that in the two years under review, 18 (50) of the babies whose mothers died didn't survive: FSBs were $7(38.9) n=18$, MSBs were 11 (61.11) $n=18$. This considered for a single year we found an increasing negative trend in 2018, 7(43.75) babies whose mothers died didn't survive compared to 2019 with 11 (55).

\subsection{Demographic and Social Characteristics of Maternal Deaths}

\begin{tabular}{|c|c|c|c|c|c|}
\hline & $\begin{array}{c}\mathbf{2 0 1 8} \\
\mathbf{( N = 1 6 )}\end{array}$ & $\begin{array}{c}\mathbf{2 0 1 9} \\
\mathbf{( N = 2 0 )}\end{array}$ & $\begin{array}{c}\text { Y FOR 2 } \\
\text { YEARS }\end{array}$ & $\begin{array}{c}\text { P-VALUE } \\
\text { [1] }\end{array}$ & P-VALUE [2] \\
\hline Age (Years) & & & & & \\
\hline$<19$ & 0 & $2(10)$ & $2(5.6)$ & & 0.4684 \\
\hline $20-24$ & $6(37.5)$ & $8(40)$ & $14(38.9)$ & & \\
\hline $25-29$ & $4(25)$ & $2(10)$ & $6(16.7)$ & 0.628 & \\
\hline $30-34$ & $4(25)$ & $6(30)$ & $10(27.8)$ & & \\
\hline$>35$ & $2(12.5)$ & $2(10)$ & $4(11.1)$ & & \\
\hline Parity & & & & & \\
\hline$<3$ & $7(43.8)$ & $10(50)$ & $17(47.2)$ & & \\
\hline $4-6$ & $7(43.8)$ & $7(35)$ & $14(38.9)$ & 0.766 & \\
\hline$>7$ & $2(12.5)$ & $3(15)$ & $5(13.9)$ & & \\
\hline Gestation period (weeks) & & & & & \\
\hline$<28$ & $1(6.3)$ & $3(15)$ & $4(11.1)$ & & \\
\hline $29-36$ & $5(31.2)$ & $7(35)$ & $12(33.3)$ & & \\
\hline$>37$ & $9(56.3)$ & $8(40)$ & $17(47.2)$ & 0.766 & \\
\hline Unknown & $1(6.3)$ & $2(10)$ & $3(8.3)$ & & \\
\hline ANC visits & & & & & \\
\hline Complete & $4(25)$ & $4(20)$ & $8(22.2)$ & & \\
\hline Incomplete & $5(31.2)$ & $5(25)$ & $10(27.8)$ & & \\
\hline Not done & $1(6.3)$ & $4(20)$ & $5(13.9)$ & 0.779 & \\
\hline Unknown & $6(37.5)$ & $7(35)$ & $13(36.11)$ & & \\
\hline
\end{tabular}

Table 1: Characteristics of Deceased Women

[1] Test of Difference in Proportion between 2018 and 2019

[2] Chi-Square Test of Trend for Both 2018 and 2019

\begin{tabular}{|l|l|l|l|l|l|}
\hline & $\begin{array}{l}\mathbf{2 0 1 8} \\
\mathbf{N = 1 6 )}\end{array}$ & $\begin{array}{l}\mathbf{2 0 1 9} \\
\mathbf{( N = 2 0 )}\end{array}$ & $\begin{array}{l}\sum_{\text {YEARS }} \text { FOR } \\
\text { YEAR-VALU }\end{array}$ & $\begin{array}{l}\text { P-VALUE [2] } \\
\text { [1] }\end{array}$ \\
\hline On admission & & & & & \\
\hline Antenatal & $6(37.5)$ & $6(30)$ & $12(33.3)$ & & \\
\hline Intrapartum & $5(31.2)$ & $5(25)$ & $10(27.8)$ & 0.243 & 0.6171 \\
\hline Post-partum & $5(31.2)$ & $9(45)$ & $14(38.9)$ & & \\
\hline At time death & & & & & \\
\hline Antenatal & $1(6.3)$ & $2(10)$ & $3(8.3)$ & & \\
\hline Intrapartum & $1(6.3)$ & $2(10)$ & $3(8.3)$ & & \\
\hline Post-partum & $13(81.3)$ & $15(75)$ & $28(77.8)$ & 0.670 & 0.0583 \\
\hline Aborting & $1(6.3)$ & $1(5)$ & $2(5.6)$ & & \\
\hline Condition on admission & & & & & \\
\hline Critical & $5(31.2)$ & 11 & $16(44.4)$ & 0.154 & 0.3458 \\
\hline Stable & $11(68.8)$ & $9(45)$ & $20(55.6)$ & & \\
\hline
\end{tabular}

\section{Table 2: Pregnancy Status}

[1] Test of Difference in Proportion between 2018 and 2019

[2] Chi-Square Test of Trend for Both 2018 and 2019

\subsection{Explanation}

The test of the difference in proportion between 2018 and 2019 for all the factors shows that there is no significant difference in proportion between the 2018 maternal mortality rates relative to the year 2019 with all the pvalues showing $<0.05$ values. 


\subsubsection{Age (Years)}

There is no significant statistical association between Age and maternal mortality, $\mathrm{p}$-value $=0.4684$ as both youths and adults show high maternal numbers at $14(36.9 \%)$ and 10 (27.8\%) respectively. At the same time the proportion of young and old women who experience maternal related deaths are relatively low at $2(0.06 \%)$ and $4(0.1 \%)$.

\subsubsection{Parity}

There is significant association between the number of times one has been pregnant and maternal mortality, pvalue $=0.0027$. Women who have had more previous pregnancies are at a lower risk of experiencing maternal death. Those who had $<3$ previous pregnancies experienced 17(47.2) deaths while those that had had more than 7 pregnancies had 5 (13.9) maternal deaths.

\subsubsection{Gestation Period (Weeks)}

There is significant association between gestation period and maternal deaths, $p$-value $=0.0006$. Women of less gestational period ( $<28$ weeks) are less likely to experience maternal related complications, $4(0.1 \%)$ as compared to those with more gestational period ( $>37$ weeks), 17(47.2).

\subsubsection{ANC Visits}

There is no significant association between ANC visits and maternal deaths, $\mathrm{p}$-value $=0.8633$, as the proportion of those who die after completing ANC visits is 8 (22.2) relative to those who do not complete ANC visit at 10(27.8). The proportion of those that do not go for ANC visits and end up experiencing maternal complications are 5 (13.9).

\subsection{Pregnancy State on Admission}

There is no significant association between on admission type and maternal deaths, $p$-value $=0.6171$. The proportion of those under Antenatal admission, Intrapartum and Post-partum are all relatively high at $12(33.3), 10(0.28)$ and $14(38.9)$ respectively.

\subsection{Condition on Admission}

Condition of the patient on admission doesn't show any statistically significant association with maternal death, pvalue $=0.3458$, as the proportion of those who are critical on admission is $16(44.4)$ but even those who die but were stable on admission is $20(55.6)$.

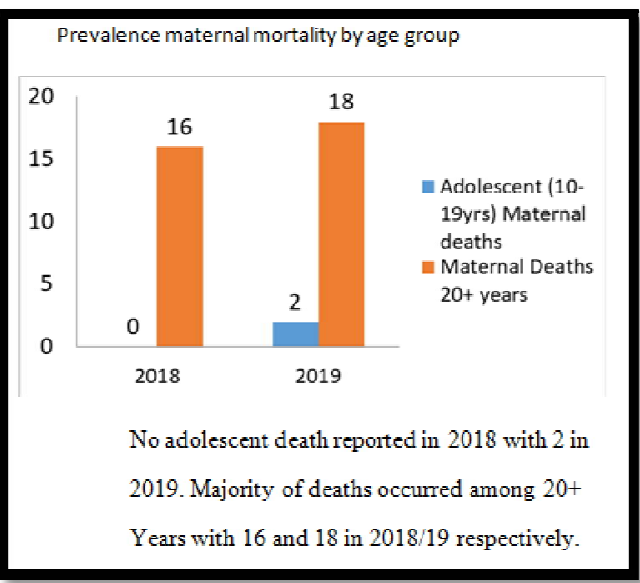

Figure 1: Prevalence Maternal Mortality by Age Group

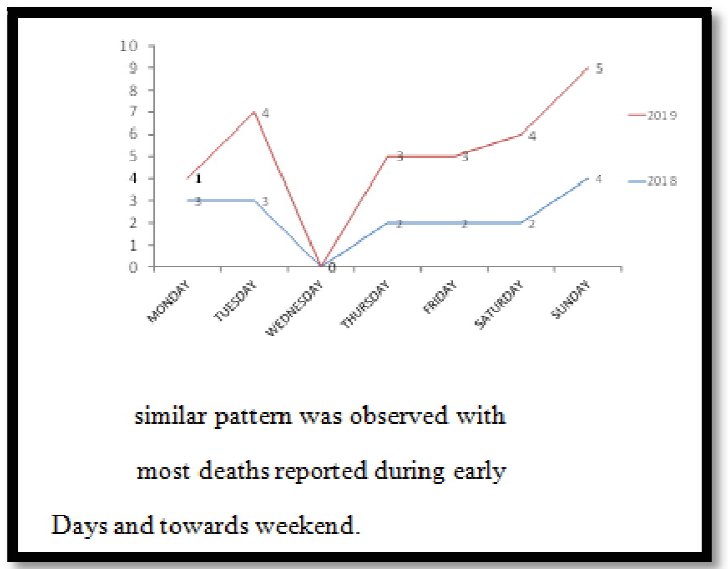

Figure 2: Disintegration of Mortality by Day Admission 


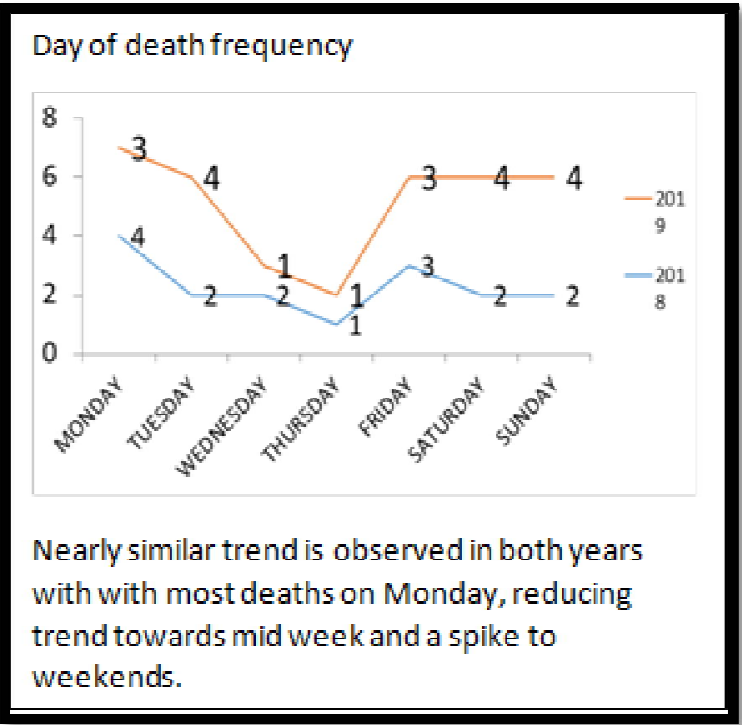

Figure 3: Day of Death Frequency

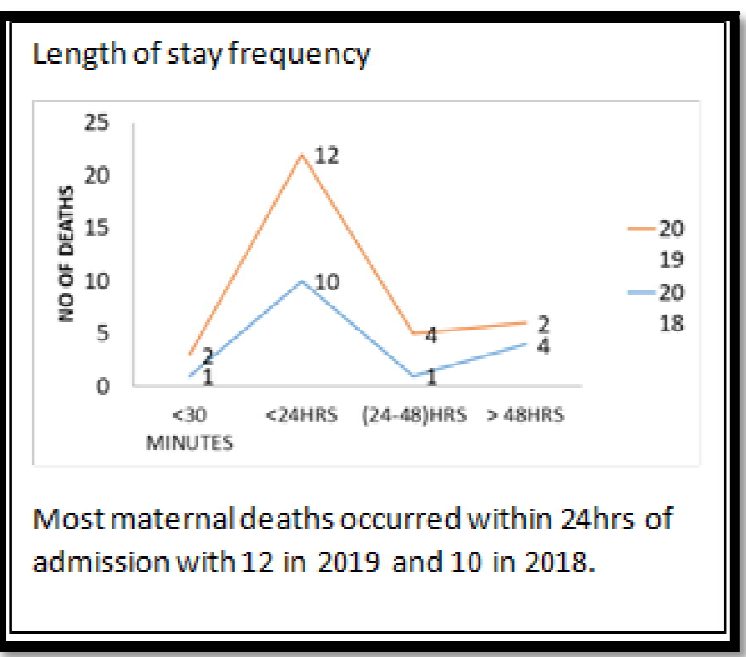

Figure 4: Length of Stay Frequency

Diagnosis as at admission

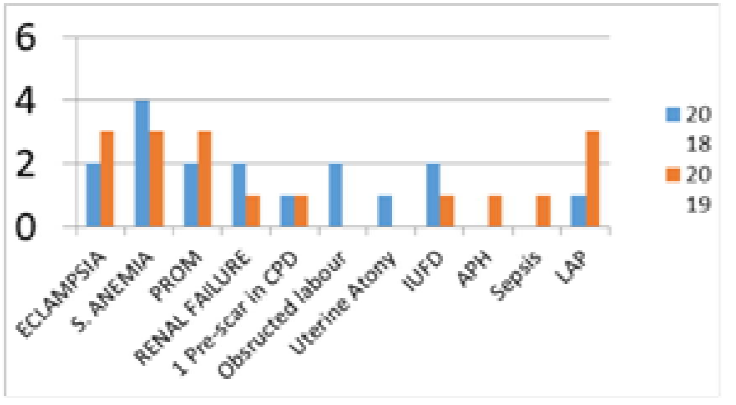

In 2018, majority of mortality's had their primary diagnoses being severe ane mia ( $3 n=16$ ) while in 2019, eclampsia, severe anaemia, PROM and LAP accounting for 3 each. In the two years, severe anemia was the primary diagnoses for $7(n=36)$ of all the deaths.

Figure 5: Diagnosis as at Admission 


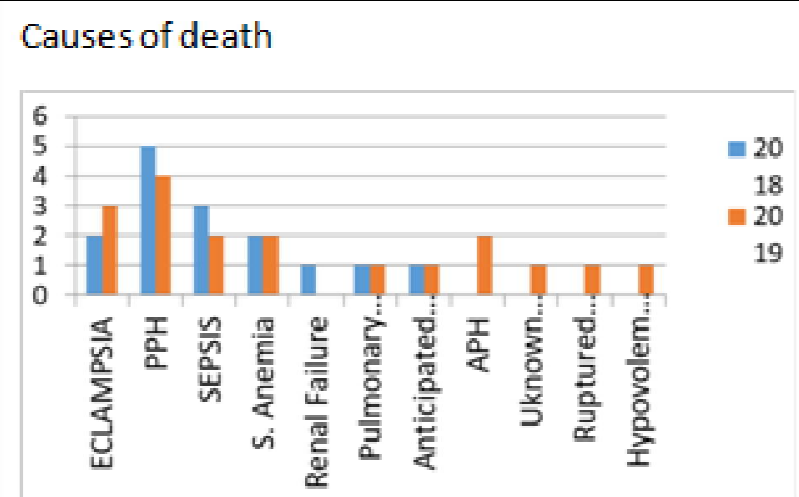

In both years, $\mathrm{PPH}$ remains the highest cause of death with 5 in 2018 and 4 in 2019.

Figure 6: Causes of Death

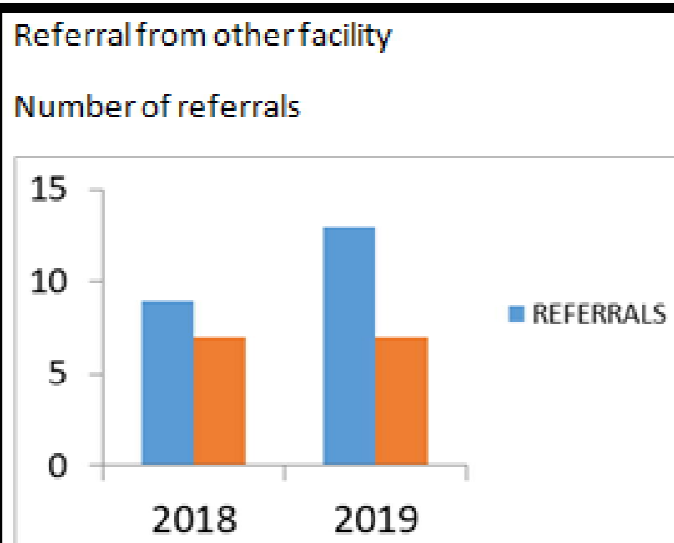

Of all the deaths in $201,9(n=16)$ were referrals with 13 $(\mathrm{n}=20)$ in 2019.

Figure 7: Referral from Other Facility

Blood/Blood products requirement

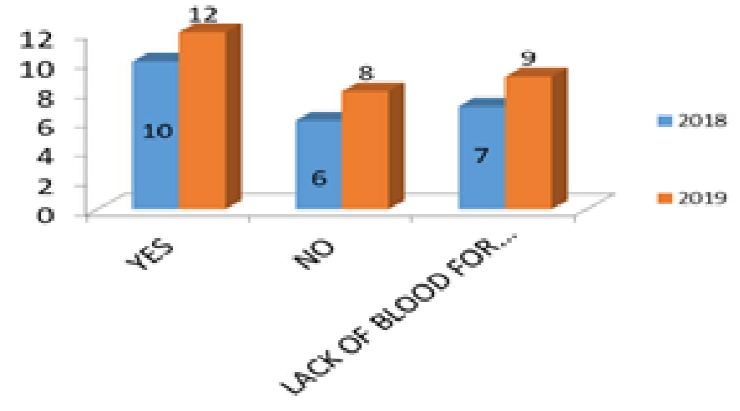

Over the two years $22(n=36)$ needed blood transfusion, 14 ( $n=36$ ) didn't require blood. of those who required blood, 7 ( $n=10)$ didn't access the product in $2018,9(n=12)$ in 2019

succumbing to lack of blood.

Figure 8: Blood/Blood Products Requirement 


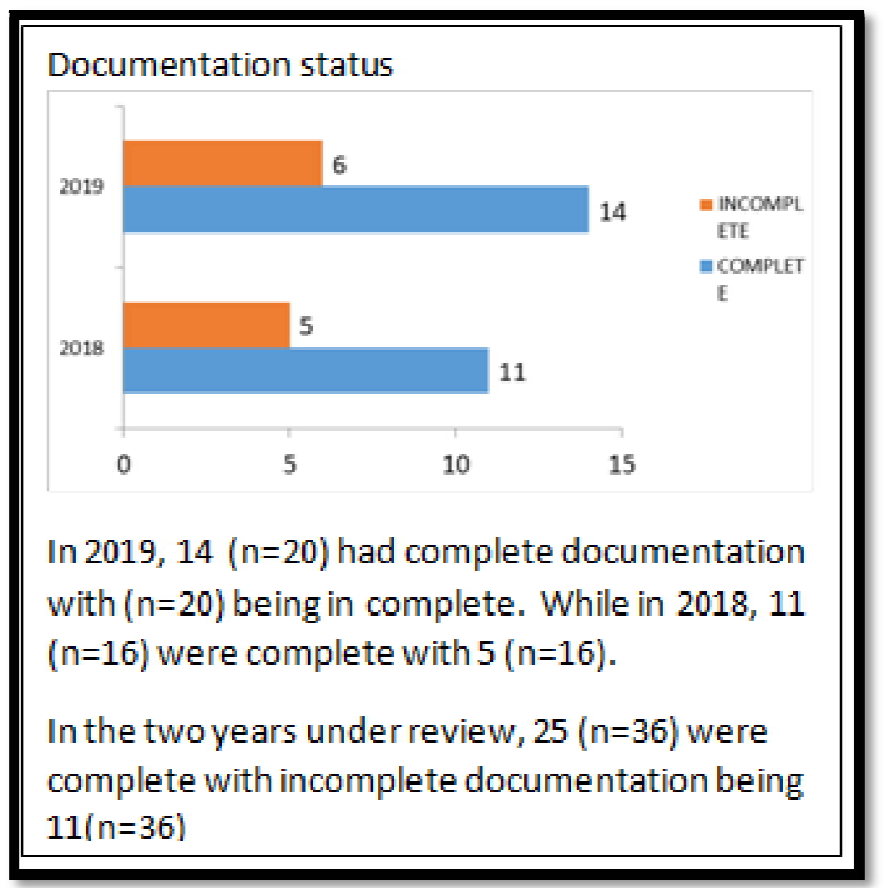

Figure 9: Documentation Status

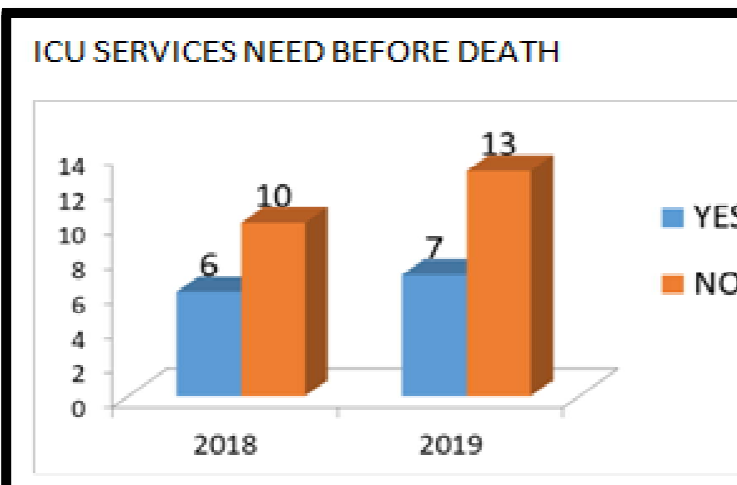

Amongst the mortalities in two years $13(n=36)$ required ICU care with $23(n=36)$ not in need of the service.

In 2018, 6 ( $n=16)$ required ICU while $10(n=16)$ weren't in need of ICU services with $7(n=20)$ in 2019 requiring ICU services while $13(n=20)$ in the same year didn't require ICU care.

Figure 10: ICU Services Need before Death

\subsection{Avoidable Factors as Opined by the MPDSR Committee during Maternal Death Audits}

- Failure to recognize danger signs at home (ANC linkage)

- Late referrals and long distance

- Insufficient staffing at maternity ward

- Inadequate skills on DVT management

- Gaps on history taking by clinicians especially on admission

- Inability by lower levels (2 \&3) to carry out ANC profiles

- Improper patients monitoring in the ward.

- Over-hydration and fast blood transfusion

- Ignorance of available services

- Poor interpersonal skills

- Insufficient blood and blood products

- Misdiagnoses

- Improper investigation

- Delayed management 


\section{Discussion}

\subsection{Age}

The risk of maternal mortality is highest for adolescent girls under 15 years old and complications in pregnancy and childbirth are higher among adolescent girls age 10-19 compared to women aged 20-24.(Mansani, Ceruti, Condemi, \& Corini, 1980).We found an irregular shift across the age groups for maternal mortality across the two year period with 2 (5.6) deaths among those aged below 19yrs. This sharply increased to 14 (38.9) for ages between 20-24yrs with a subsequent reduction to 6 (16.7) for 25-29yrs and an increase again to 10 (27.8) for 30-34yrs and a slight maternal mortality of 4 (11.1) for women above 35yrs. This is inconsistent with WHO report of 2019 that the risk of maternal mortality is highest for adolescent girls under 15 years old and complications in pregnancy and childbirth are higher among adolescent girls age 10-19 (compared to women aged 20-24). (WHO, 2019).Ann K. Blanc reported similar finding of an irregular shift across the various age groups with the maternal mortality ratio starts low and rises steeply and nonlinearly after age 30; the MMR curve becomes progressively steeper as age advances. The ratio starts just above 400, dips to 319 among women age 20-24, and then rises to 1351 in the oldest age group.(Blanc, Winfrey, \& Ross, 2013) This study finds no significant statistical association between age and maternal mortality implying that age isn't a risk factor for maternal mortality. This is consistent with other studies that found that the risk of mortality associated with adolescent pregnancy is only about a third higher than that of women aged 20-24 years (260 [uncertainty 100-410] vs190 [120-260] maternal deaths per 100000 live births), and therefore not as high as previously believed.(Restrepo-Méndez \& Victora, 2014). Cumulatively 14 women of above 30yrs died during the period under review. This reviewed with other findings reveals no increased risk for maternal mortality as shown by in a review by Lancet team which found that women older than 35 years had the highest maternal mortality ratios. And although this pattern has been described before, no previous study has reported such convincing data from such a wide range of countries. (Blanc et al., 2013).

\subsection{Parity}

It has been suggested that multiparty is associated with a higher level of risk (maternal death) than primi-parity. (Hogberg \& Wall, 1986). Obstetric hemorrhage and complications of labor were more common among Para 2-4 individuals.(Amatare\& Dinyain, G Olutoyin Omoniyi-Esan, Olaejirinde O Olaofe, Donatus Sabageh, Akinwumi O Komalafe, 2013). This can be inferred to our study that found significant association between the number of times one has been pregnant and maternal mortality, $\mathrm{p}$-value $=0.0027$. Women who have had more previous pregnancies are at a lower risk of experiencing maternal death. Those who had $<3$ previous pregnancies experienced 17(47.2) deaths while those that had had more than 7 pregnancies had 5 (13.9) maternal deaths. This may not be quite conclusive due to the small sample size and lack of similar studies in other reviews.

\subsection{Gestation Period (Weeks)}

There is significant association between gestation period and maternal deaths, $p$-value $=0.0006$. Women of less gestational period ( $<28$ weeks) are less likely to experience maternal related complications, $4(0.1 \%)$ as compared to those with more gestational period ( $>37$ weeks), 17(47.2).However, amongst other peer-reviewed articles of previous studies, no study was found to link gestational age with increased risk of maternal death. Even in similar studies such as 2009-2013 maternal death audit review in Rwanda. (Sayinzoga et al., 2016) and maternal mortality review in a Nigerian hospital.(Olopade, F.E and Lawoyin, 2008)where maternal death reviews analysis were made, no such inferences were made. This study therefore opens a new field for future research.

\section{Limitations}

Small sample size.

\section{Conclusion}

There's negative increasing trend of maternal mortalities overtime

Maternal death reviews have not led to reduction in maternal deaths in the referral hospital

Post-partum Haemorrhage remains a leading cause of death across the two years under review.

\section{Recommendation}

\subsection{Future Research}

We recommend a research investigation on an association between gestational age and maternal mortality with larger sample.

Gap study analysis should be done between MDR and implementation of recommendations made in such reviews. Maternal health services including blood transfusion and surgical services should be introduced in lower service units as most of deaths reported originated from other facilities.

\section{Compliance with Ethical Standards}

- Conflicts of Interest: The authors of this paper have no conflict of interest in this work

- Funding/Support: Afya Halisi and county Government of Migori supported these review meetings.

- Ethics Approval: Permission for this study and publication was issued by the director-medical services. 
- Acknowledgements:MPDSR committee of Migori county Referral Hospital, AfyaHalisi and Office of the director medical services- Migori County.

\section{References}

i. Amatare, \&Dinyain, G OlutoyinOmoniyi-Esan, Olaejirinde O Olaofe, DonatusSabageh, Akinwumi O Komalafe, 0. S. 0. (2013). Autopsy-certified maternal mortality at Ile-Ife Nigeria. (December). https://doi.org/10.2147/IJWH.S39863

ii. Bale, J. ., Stoll, B. J., \& Lucas, A. O. (2003). Reducing Maternal Mortality and Morbidity - Improving Birth Outcomes. Retrieved from https://www.ncbi.nlm.nih.gov/books/NBK222105/

iii. Blanc, A. K., Winfrey, W., \& Ross, J. (2013). New Findings for Maternal Mortality Age Patterns: Aggregated Results for 38 Countries. PLoS ONE, 8(4). https://doi.org/10.1371/journal.pone.0059864

iv. Hofman, J. J., \& Mohammed, H. (2014). Experiences with facility-based maternal death reviews in northern Nigeria. International Journal of Gynecology and Obstetrics, 126(2), 111-114. https://doi.org/10.1016/j.ijgo.2014.02.014

v. Hogberg, U. L. F., \& Wall, S. (1986). Age and parity as determinants of maternal mortality impact of their shifting distribution among parturients in Sweden from 1781 to 1980. 64(1), 85-91.

vi. Koltai, J., \&Schieman, S. (2015). Policy Brief. Journal of Health and Social Behavior, 56(2), 179. https://doi.org/10.1177/0022146515584605

vii. Mansani, F. E., Ceruti, M., Condemi, V., \&Corini, L. (1980). Adolescent pregnancy. ActaBiomedica de l'AteneoParmense, Vol. 51, pp. 365-369.

viii. Olopade, F.E and Lawoyin, T. O. (2008). Maternal Mortality in a Nigerian Maternity Hospital. 11(June), 267273.

ix. Restrepo-Méndez, M. C., \&Victora, C. G. (2014). Maternal mortality by age: Who is most at risk? The Lancet Global Health, Vol. 2, pp. e120-e121. https://doi.org/10.1016/S2214-109X(14)70007-5

x. Sayinzoga, F., Bijlmakers, L., Dillen, J. Van, Mivumbi, V., Ngabo, F., \&Velden, K. Van Der. (2016). Maternal death audit in Rwanda 2009 - 2013: a nationwide facility-based retrospective cohort study. 1-8. https://doi.org/10.1136/bmjopen-2015-009734

xi. WHO, UNICEF, UNFPA, World Bank Group, and U. N. P. D., \& Group, M. M. E. I.-A. (2015). Maternal mortality in 1990-2015. 1-5.

xii. WHO. (n.d.-a). Maternal deaths fell 44\% since 1990 - UN. Retrieved from https://www.who.int/newsroom/detail/12-11-2015-maternal-deaths-fell-44-since-1990-un

xiii. WHO. (n.d.-b). Maternal mortality. Retrieved from https://www.who.int/news-room/factsheets/detail/maternal-mortality

xiv. WHO. (2020). WHO | SDG 3: Ensure healthy lives and promote wellbeing for all at all ages. Who. 